\title{
Role for integrin-linked kinase in mediating tubular epithelial to mesenchymal transition and renal interstitial fibrogenesis
}

Yingjian Li, Junwei Yang, Chunsun Dai, Chuanyue Wu, and Youhua Liu

Original citation: J. Clin. Invest. 112:503-516 (2003). doi:10.1172/JCI200317913.

Citation for this corrigendum: J. Clin. Invest. 113:491 (2004). doi:10.1172/JCI200417913C1.

During the preparation of this manuscript for publication, errors were introduced into Figure 1 . The corrected figure and legend appear below. These changes do not affect the conclusion of the paper.

Additionally, the grant "DK-54944" should read as "DK-54922". The authors regret these errors.

a

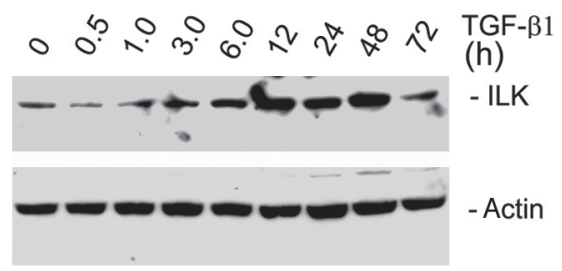

b

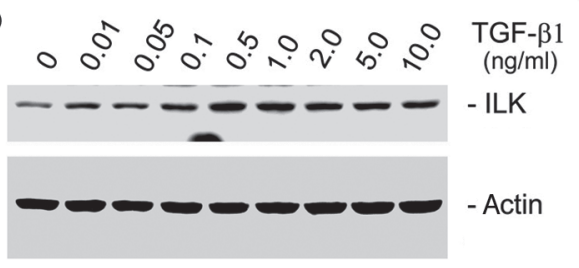

c

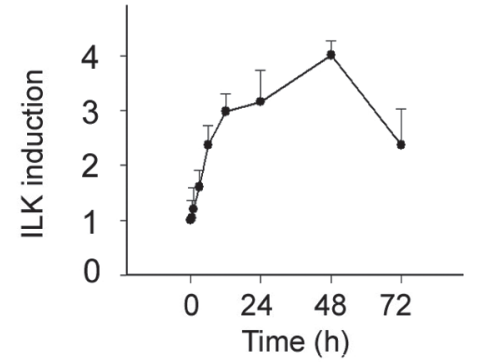

d

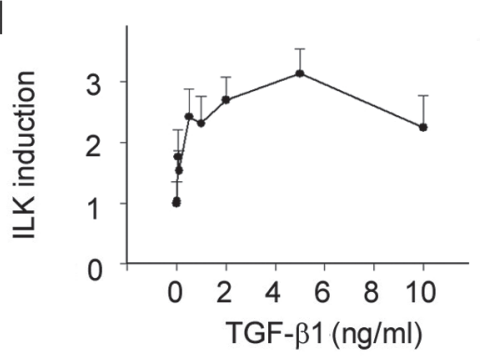

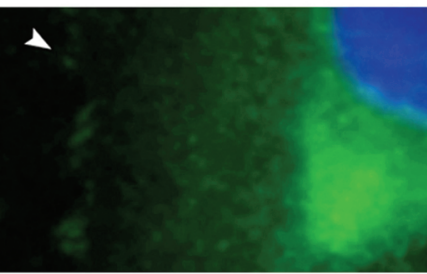

f

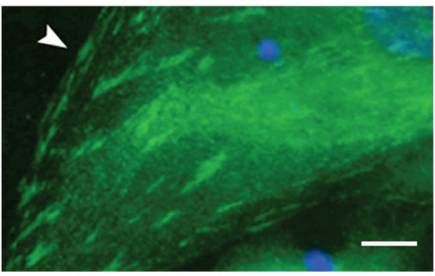

\section{Figure 1}

TGF- $\beta 1$ induces ILK expression in renal tubular epithelial cells. (a-d) Western blot analyses show that TGF- $\beta 1$ induced ILK protein expression in a time- and dose-dependent manner. HKC cells were incubated with either the same concentration of TGF- $\beta 1$ ( 2 ng/ml) for various periods of time as indicated ( $\mathbf{a}$ and $\mathbf{c}$ ) or increasing amounts of TGF- $\beta 1$ for 24 hours (b and $\mathbf{d}$ ). Cell lysates were immunoblotted with Ab's against ILK and actin, respectively. (a and $\mathbf{b}$ ) Representative Western blots. (c and $\mathbf{d}$ ) Graphic presentation of relative ILK abundance (fold induction) normalized to actin. Data are presented as mean \pm SEM of three independent experiments. (e and $\mathbf{f}$ ) Immunofluorescence staining shows the localization of ILK in control (e) or TGF- $\beta 1$-treated HKC cells (f). Arrowheads indicate positive ILK staining. Scale bar: $5 \mu \mathrm{m}$. 